\title{
Pratiques pastorales Mbororo et trypanosomoses bovines dans une zone de savanes humides de Centrafrique
}

\author{
F. D’Amico ${ }^{1,2}$, J. M. Poussinga 2 , C. Le Masson ${ }^{3}$, A. Le Masson ${ }^{4}$, D. Cuisance ${ }^{1}$
}

D'AMiCO (F.), POUSSinga (J.M.), LE MASSON (C.), LE MASSON (A.), CUISANCE (D.). Pratiques pastorales Mbororo et trypanosomoses bovines dans une zone de savanes humides de Centrafrique. Revue Élev. Méd. vét. Pays trop., 1995, 48 (2) : 203-212

L'étude a porté sur l'agencement de l'espace pastoral chez les éleveurs Mbororo de Centrafrique et les mouvements de leurs zébus à robe acajou. Outre le campement des éleveurs, cet espace est divisé en trois compartiments principaux : l'aire de repos, l'abreuvoir et le pâturage sillonné de sentiers. Son utilisation repose sur une ségrégation spatiale et temporelle des déplacements des animaux. Dans le contexte particulier des savanes humides du centre de la République centrafricaine, où l'espèce riverainc Glossina fuscipes fuscipes Newst. 1910 est le vecteur principal des trypanosomoses bovines, les auteurs montrent qu'une connaissance approfondie des pratiques pastorales apporte de nouveaux éléments à la compréhension de l'épidémiologie du nagana. Ainsi, la conduite différentielle des veaux par rapport aux adultes est-elle vraisemblablement un facteur épidémiologique capital.

Mots clés : Bovin - Zébu - Trypanosomose - Glossina fuscipes fuscipes - Fpidémiologie - Pastoralisme - Société pastorale - Parcours Transhumance - République Centrafricaine.

\section{INTRODUCTION}

L'installation des Peul Mbororo dans les savanes humides de Centrafrique date des années 1920-1930 (5, 19, J. Desrotour, com. pers.). En langue fulfuldé, wadahoundé désigne les trypanosomoses animales, dues à $T$. brucei, $T$. congolense et $T$. vivax. L'ampleur des pertes dues à ces maladies en République centrafricaine (RCA) grève considérablement le développement de l'élevage $(9,13,22,41)$. Afin d'amener l'incidence de cette affection à un niveau compatible avec l'élevage, des solutions en rapport direct avec l'épidémiologie de ces protozooses doivent être proposées $(8,15,16)$. Ceci implique une connaissance approfondie des différents acteurs épidémiologiques : les trypanosomes parasites, les vecteurs possibles et l'hôte final, le zébu Mbororo à robe acajou (7).

Les relations existant entre ces organismes sont encore méconnues. Les résultats obtenus au cours des principaux travaux réalisés en RCA témoignent incontestable-

1. CIRAD-EMVT, c/o Centre ORSTOM de Montpellier, Département Santé, 911 avenue Agropolis, BP 5045, 34032 Montpellier Cedex 1, France.

2. Centre ORSTOM de Bangui, B.P. 893, Bangui, République Centrafricaine.

3. C/o A. Le Masson

4. CIRAD-EMVT, BP 5035, 34032 Montpellier, Cedex 1, France

Reçu le 2.6.1994, accepté le 19.7.1995. ment du fait que Glossina fuscipes fuscipes Newstead, 1910 est le vecteur majeur des trypanosomoses bovines dans l'ensemble du pays $(8,9,13)$. Selon ces auteurs, la contamination des animaux s'effectue essentiellement au niveau des points d'abreuvement et ce toute l'année. Les travaux récents (10) utilisant le concept de risque trypanosomien $(21,35,38)$ comme marqueur épidémiologique, confirment que $G$. $f$. fuscipes est bien le vecteur majeur mais que le comportement vectoriel de cette espèce n'explique pas de manière satisfaisante et correcte les valeurs élevées des prévalences trypanosomiennes bovines, tout au long de l'année.

Ces incertitudes découlent du manque de connaissance des mécanismes épidémiologiques intimes qui règlent la transmission des parasites aux bovins. Parmi tous les facteurs intervenants, le plus méconnu est sans doute l'éthologie des zébus. Celle-ci étant fortement tributaire des contraintes imposées par les éleveurs, on l'incluera sous le terme de "pratiques pastorales" dans la suite de cet article. Bien que les pratiques pastorales soient souvent incriminées dans la transmission de la maladie (29, $33,34,36$ ), elles n'ont encore été que très peu étudiées. Quelques observations ont été réalisées chez les taurins d'Afrique occidentale en particulier $(39,40)$ mais une seule, à la connaissance des auteurs, concerne les zébus (27). L'objectif du présent article est de pallier ces lacunes et de montrer qu'en Centrafrique au moins, la connaissance des mouvements des zébus et des pratiques pastorales est indispensable à la compréhension de l'épidémiologie des trypanosomoses bovines.

\section{MATÉRIELet MÉTHODES}

L'étude a eu tieu en 1991 et 1992 chez cinq éleveurs Peul Mbororo Djafoun de la commune d'Ouro-Djafoun (Bambari, préfecture de la Ouaka, RCA). Le secteur appartient au domaine soudano-oubanguien (37) et reçoit annuellement 1350 à $1590 \mathrm{~mm}$ de pluie (14) Deux types d'élevages ont été retenus, l'un sédentaire'à titre pratiquement expérimental (1 élevage) et l'autre semi-transhumant traditionnel (4 élevages).

Au cours de l'année, des visites mensuelles chez les différents éleveurs choisis ont permis de constituer une documentation de première main concernant l'organisation spatiale de l'activité pastorale et les mouvements des troupeaux. Les infections trypanosomiennes chez le bétail ont été suivies au cours de visites trimestrielles. A 
partir d'échantillons sanguins prélevés à la veine auriculaire ou à la veine caudale, la présence de trypanosomes a été appréciée par deux méthodes différentes : i) cxamen microscopique sur frottis, sur goutte épaisse et après centrifugation en tube hématocrite $(18,28)$ et ii) détection sérologique au moyen d'anticorps monoclonaux (technique ELISA) d'antigènes circulants trypanosomiens $(30,31)$.

L'élevage sédentarisé, à caractère expérimental, offrant une meilleure disponibilité du bétail, a permis d'accumuler des informations précises sur les parcours et sur la distribution horaire des activités du bétail en saison des pluies. La majorité des résultats présentés est tirée de cette observation. Dans la mesure du possible, les termes précis se rapportant à l'élevage peul sont transcrits en langue fulfuldé et indiqués en italique.

\section{RÉSULTATS et DISCUSSION}

\section{Les types de mobilités pastorales}

Selon une conception habituelle, les pasteurs Peul, essentiellement mobiles, ne s'inscrivent pas durablement dans leurs espaces pastoraux successifs et ne les délimitent pas matériellement. En fait, la position des campements et du bétail n'est jamais aléatoire dans les savanes. Elle est régie par des habitudes ou même des règles qui relèvent souvent de compromis entre des contraintes contradictoires.

\section{L'organisation fondamentale de l'espace pastoral}

L'espace pastoral désigne l'ensemble des sites occupés par l'éleveur et sa famille ainsi que ceux fréquentés quotidiennement par son bétail (figure 1). La famille de l'éleveur est regroupée au niveau du campement fait de huttes de bois et de paille, dont le nombre dépend de l'importance de la famille. Selon le moment de la journée, le bétail se trouve à l'aire de repos (DuuDaal nagge ou pellel na'i), aux points d'abreuvement (regorde) ou au pâturage $(h u D o)$. L'aire de repos est une aire dégagée où est rassemblé le troupeau pour la traite, les opérations de détiquage et le contrôle sanitaire des animaux. L'intégration et la disposition géographique de tous ces éléments varient beaucoup et s'adaptent généralement à la morphologie du terrain. Le campement est situé le plus souvent sur un interfluve (figure 1), à distance raisonnable de la rivière, pour éviter les désagréments liés à l'entomofaune, mais assez proche de façon à éviter de trop longs déplacements liés à l'approvisionnement en eau pour les besoins domestiques. En général, l'aire de repos du bétail est à l'écart du campement mais reste visible de celui-ci. Elle peut être divisée en plusieurs unités, chacune accueillant le troupeau d'un des membres de la famille. Le nombre et l'éloignement des points d'abreuvement ainsi que l'étendue du pâturage dépendent de nombreux facteurs. Tous ces lieux sont reliés les uns aux autres par plusieurs sentiers, certains empruntés le plus souvent par les gons, d'autres par le bétail.

\section{Les déplacements chez les Peul Mbororo}

Nomades par tradition, les Mbororo et leurs zébus se déplacent plus ou moins toute leur vie. Trois types de déplacements peuvent être distingués :

\section{Migrations "historiques}

Ce sont les déplacements d'une amplitude comprise entre 10 et $500 \mathrm{~km}$, qui concernent l'ensemble de la famille peul, accompagnée de tout son cheptel $(24,25)$. Ils s'effectuent sur des distances parfois considérables et correspondent généralement à des changements de région ou de pays, consécutifs à des échecs ou à des difficultés rencontrées tant sur le plan sanitaire que politique (5). Comme exemple, on peut citer l'entrée des Peul en Centrafrique, leurs installations successives à Bouar et Bossembélé à l'ouest et à Bambari au centre-est, les tentatives jusqu'à Mobaye et même Djema à l'extrême est du pays. Loin d'être des conquêtes progressant de manière sûre, ces déplacements sont oscillants, avec une succession d'avancées et de reculs qui s'enchaînent (J. Desrotour, com. pers.).

\section{Mouvements saisonniers}

Il s'agit des transhumances, déplacements de plus faible envergure puisque compris entre 10 et $150 \mathrm{~km}$, amenant les troupeaux dans de nouveaux pâturages à chaque saison sèche (20). Ces déplacements peuvent impliquer toute la population du campement, ou seulement une partie de la famille ; les autres personnes restent alors sur place en compagnie de vaches laitières et de leurs veaux. Les distances parcourues sont plus longues dans l'Ouest centrafricain que dans la région de Bambari où elles n'atteignent que quelques kilomètres à quelques dizaines de kilomètres. Ces mouvements commoncent en général en octobre et s'étalent jusqu'en mai ou juin. Ils ont été bien étudiés dans le contexte centrafricain $(5,6,25)$.

\section{Déplacements quotidiens}

Encore peu connus (27), ils concernent les différents circuits de pâturage effectués chaque jour par le bétail entre l'aire de repos, le point d'abreuvement et le pâturage. La distance quotidienne moyenne parcourue est de 5 à $8 \mathrm{~km}$ en saison des pluies, et souvent plus en saison sèche. C'est ce type de mobilité du bétail que l'enquête a étudié dans son ampleur et ses modalités, pendant la saison des pluies.

\section{Les circuits quotidiens des animaux}

Chaque jour, le bétail quitte l'aire de repos pour aller s'abreuver et paître. Le parcours suivi n'est ni aléatoire ni immuable. Il dépend de l'âge et du statut des animaux. 


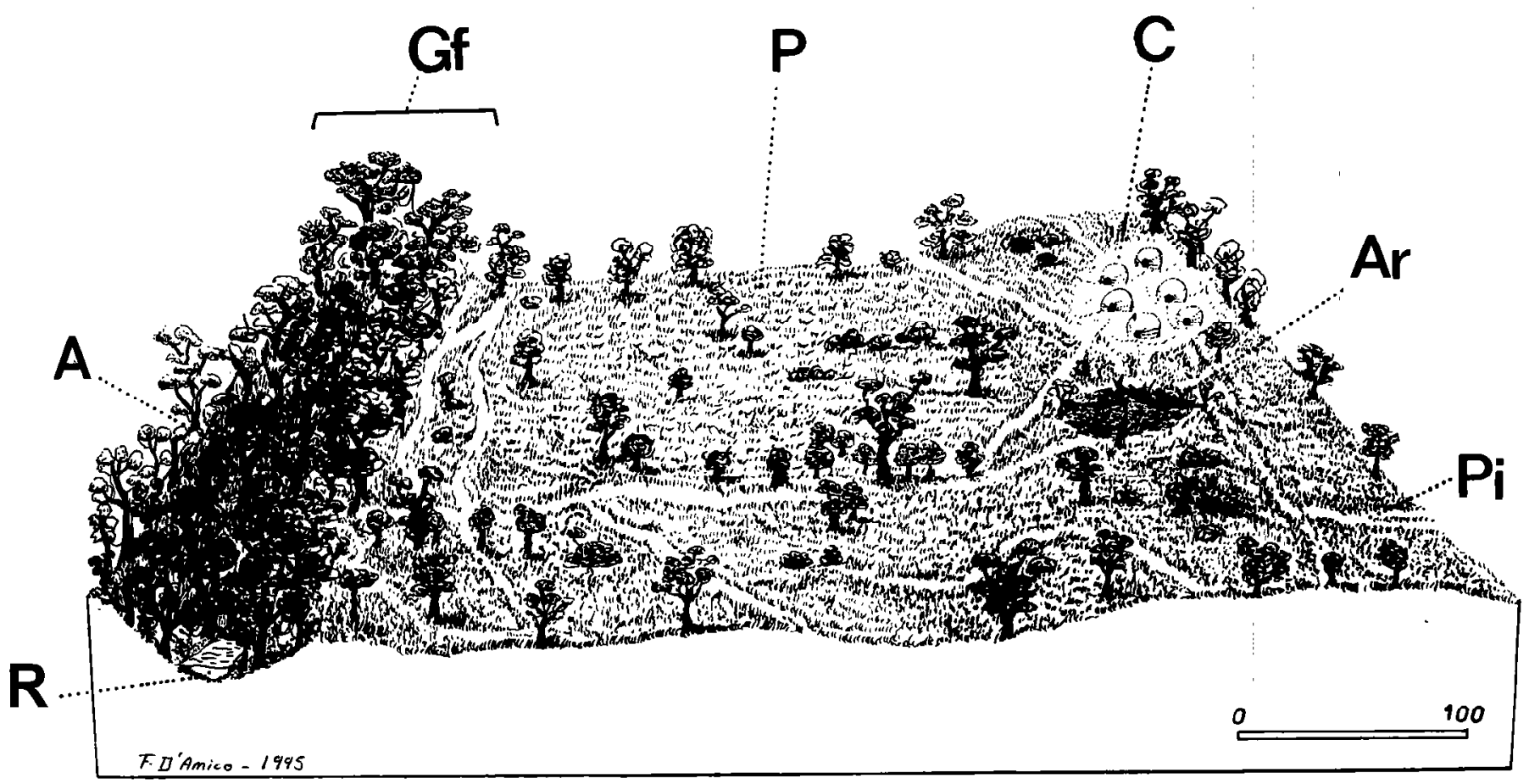

Figure 1 : Les principaux éléments de l'espace pastoral Mbororo. Le campement (C), constitué de plusieurs huttes rondes de bois et de paille, est installé en gènéral sur une hauteur, à une distance variable de la galerie forestière (Gf). Celle-ci est un bande forestière dans laquelle circule la rivière (R). Le bétail s'abreuve en un point à peine aménagé de celle-ci et nommé abreuvoir $(A)$. Le pâturage $(P)$ est la vaste surface de savane arborée utilisée pour l'alimentation des zébus et sillonné de pistes (Pi) suivies par le bétail. A proximité du campement, se situe l'aire de repos (Ar) qui est une simple surface non clòturée et dérnuée de végétation en raison du piétinement répété des zébus. L'aire de repos est le lieu de rassemblement du troupeau et elle est utilisée par l'éleveur pour les opérations de traite et de contrôle sanitaire des animaux, en particulier le détiquage.

La composition d'un troupeau de zébus Mbororo évolue au cours de l'année. Suivant les zones d'élevage, les gestations se déroulent à des périodes différentes dans l'année puisque les dates de fécondation sont souvent tributaires des transhumances (A. Le Masson, données non publiées). Dans la zone étudiée, les naissances ont lieu au cœur de la saison sèche, entre décembre et mars généralement. A partir de cette période, les soins donnés au troupeau et sa conduite vont changer légèrement, en rapport avec la croissance des veaux jusqu'au sevrage, qui intervient dans la fin de leur première année. Pendant la saison sèche, correspondant aux premiers mois de leur vie, les veaux de moins de 6 mois sont tenus attachés par une corde particulière (dangol) dans l'aire de repos. Délivrés seulement au moment où les mères rentrent du pâturage, les veaux sont autorisés à téter quelques instants pour stimuler la descente du lait et sont chassés aussitôt après ; la traite effectuée, ils sont laissés sous la mère pour la fin de leur alimentation. A partir de 5 à 6 mois, les veaux sont libérés pendant la journée et leur calendrier quotidien se calque alors sur celui observé chez les animaux adultes pendant la journée.

Chaque catégorie de bétail (les veaux d'un côté, le reste du troupeau de l'autre) évolue quotidiennement selon un cycle bien défini, qui commence le matin lorsque les bêtes sont poussées par l'éleveur ou l'un de ses fils jusqu'au point d'abreuvement. Le temps consacré à l'abreuvement est généralement très bref, quelques minutes suffisant à cette opération. Les Mbororo disent qu'ils "poussent" (yerba) les animaux en direction du pâturage choisi. Par la suite, une fois repus, les vaches allaitantes toujours devant, les zébus rentrent par petits groupes à l'aire de repos pour s'y arrêter ou pour continuer à paître aux environs du campement. Parfois, sur le chemin du retour, surtout lorsque celui-ci passe par un point d'eau, les animaux peuvent s'abreuver à nouveau. Dans la soirée, le retour du bétail (jaingol) aux abords du campement est spontané ou accéléré par l'éleveur qui va à la recherche de ses animaux. Avant la tombée de la nuit, le troupeau est rassemblé à l'aire de repos, autour d'un petit feu de feuilles vertes destiné à éloigner les insectes piqueurs. De façon générale, les animaux adultes partent la nuit au pâturage, jusqu'à 3 ou 4 heures du matin, tandis que les veaux restent attachés. Ce pâturage de nuit, jamais observé chez les veaux de moins d'un an, est dénommé soggal ou wambbrinde. Au petit matin, avant que le soleil ne se lève, les femmes procèdent à la traite tandis que les hommes examinent les bêtes et dispensent les soins nécessaires (désinfection des plaies, détiquage...).

Le temps passé à chaque endroit varie légèrement chaque jour. Globalement, au fur et à mesure que la saison des pluies s'avance, le cycle quotidien se modifie quelque peu (figure 2). En mai, à la sortie de la saison 


\section{F. D'Amico J.M. Poussinga C. Le Masson A. Le Masson D. Cuisance}
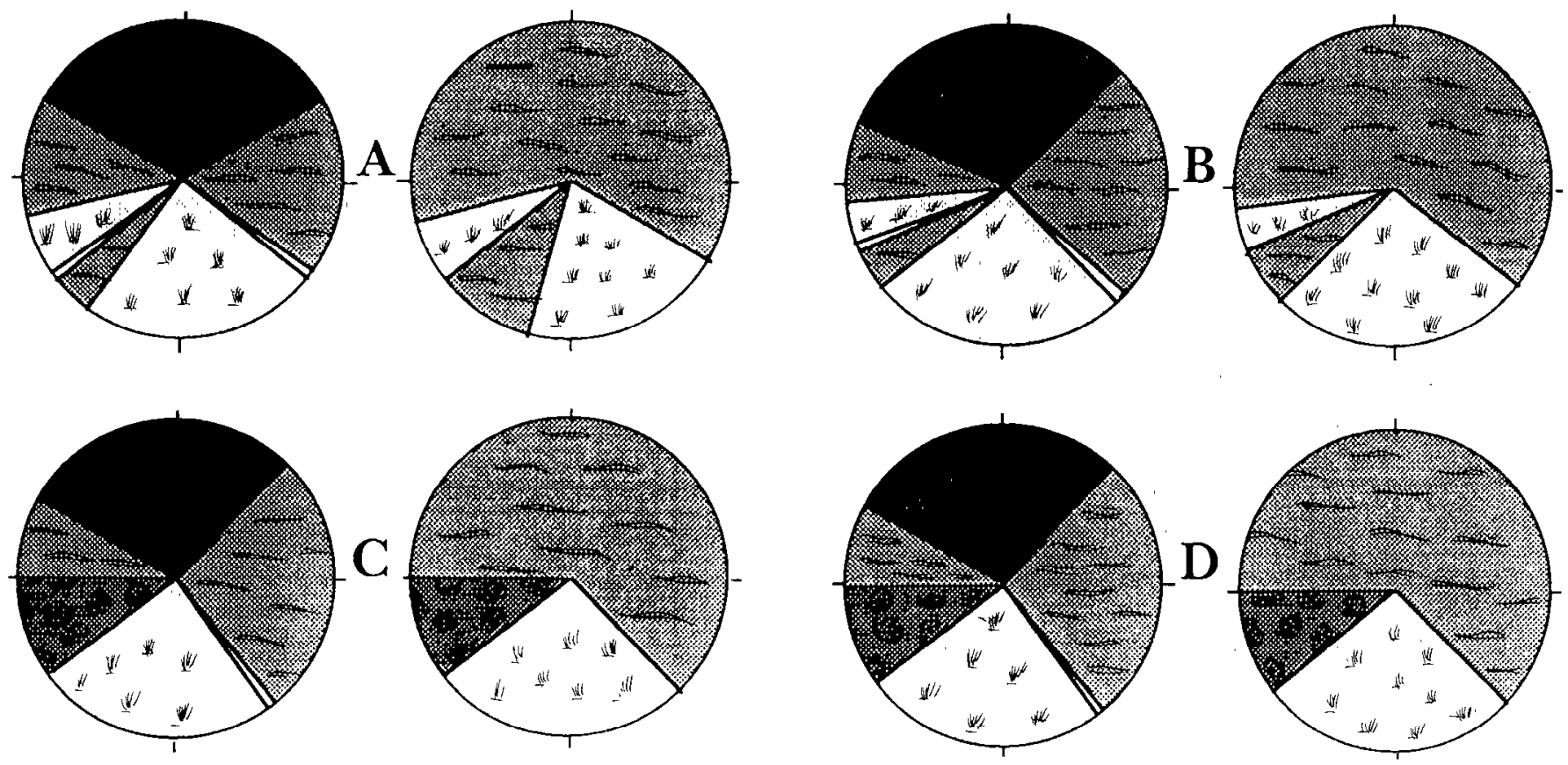

Adultes

Veaux

Adultes

Veaux

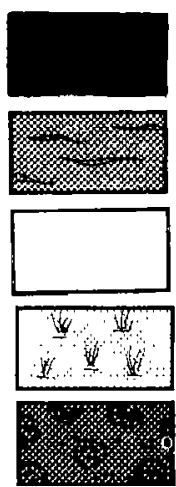

pature nocturne

sciont a l'are de repors

ahrous ament

pàture diumc

pâture atutour de liare de repos

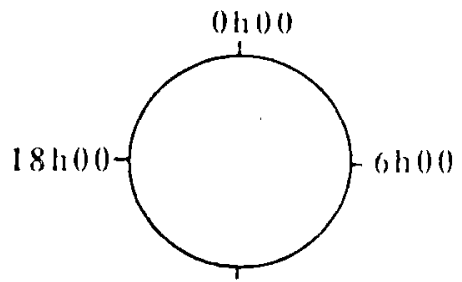

$12 \mathrm{~h} 00$

Figure 2 : "Cycle d'activitée" journalier des zébus Mbororo (veaux et adultes) et variations au cours de la saison des pluies. (A : mai $1992 ; B ; j u i n$ 1992;C : juillet $1992 ; D$ : août 1992). Ce cycle comporte deux séquences d'abreuvement et de pâture en mai et juin, et une seule en juillet et août. Noter l'absence de pâture nocturne chez les veaux, qui restent attachés à l'aire de repos. Les périodes d'abreuvement chez les veaux, mal connues, n'ont pas été représentées.

sèche, le troupeau étudié avait deux périodes d'alimentation dans la journée, une le matin et une le soir, avec à chaque fois un passage au point d'abreuvement (fig. 2 : A). Juin et juillet constituent une période de transition (fig. 2 : B, C) durant laquelle l'abreuvement du soir est progressivement supprimé, alors que le pâturage de fin d'après-midi, bien individualisé initialement, devient moins prépondérant et se transforme en un séjour localisé autour de l'aire de repos. Ce fait s'observe pour toutes les classes d'âge. En août, au cœur de la saison des pluies, le pâturage s'effectue en une seule fois et ne s'accompagne que d'un passage au point d'abreuvement (fig. 2 : D).
Si dans leur composante temporelle les rythmes d'activité des veaux et des adultes se ressemblent, il n'en va pas de même pour leur composante spatiale. En effet, pendant la saison des pluies, les veaux réunis en un petit troupeau (nyalbi) suivent un parcours différent de celui du gros du troupeau (sefre) et s'abreuvent en un point différent. Cette affirmation doit être nuancée car, en début de saison des pluies, en fin d'après-midi, les veaux et le reste du troupeau peuvent se regrouper (figure $3 A$ ). En ce qui concerne le troupeau adulte, bien que peu d'informations précises aient pu être recueillies, il semble que les parcours diurne et nocturne ne se recouvrent pas. 


$$
\frac{Y}{Y}=
$$




\section{Pratiques pastorales et infections trypanosomiennes}

Une des priorités de notre recherche est d'identifier un lien entre les caractéristiques épidémiologiques de la maladie et la variabilité observée dans la circulation du bétail à l'échelle d'une journée, d'une saison, d'une année, d'une décennie ou même d'un siècle, entre diverses régions.

\section{Conséquences prévisibles des déplacements chez les Peul}

Parmi les trois types de mobilité rencontrés chez les Peul, les éleveurs soupçonnent avant tout les transhumances de favoriser certaines trypanosomoses. Les trajets de transhumance sont parfois longs, complexes et bien souvent indéterminés par avance. Leur effet sur l'épidémiologie des trypanosomoses n'est pas facile à cerner, puisqu'il faudrait connaître la situation glossinienne et trypanosomienne de chaque galerie forestière fréquentée au cours des périples pastoraux. Toutefois, une étude comparative de la prévalence et de l'incidence des trypanosomoses chez le bétail avant et après les transhumances de saison sèche devrait apporter rapidement quelques informations qui permettront d'infirmer ou de confirmer les propos des éleveurs.

De façon analogue, les grandes migrations historiques conduisent vraisemblablement à un brassage complexe des parasites et, de ce fait, pourraient expliquer la grande variété de formes épidémiologiques (sporadique, enzootique, épizootique, mixte) rencontrées en Centrafrique. Toutefois, les mouvements quotidiens, de plus faible amplitude, sont plus aisés à étudier et leur influence sur les infections trypanosomiennes paraît plus facile à appréhender.

\section{Impact des pratiques pastorales au quotidien}

D'ores et déjà, l'étude montre que trois grands faits peuvent définir la pratique pastorale Mbororo au quotidien :

\section{L'organisation fonctionnelle de l'espace pastoral}

Elle se traduit par un découpage de l'espace pastoral en plusieurs domaines fondamentaux (campement de l'éleveur, aire de repos du bétail, abreuvoirs, sentiers et pâturages). Chacun d'entre eux se caractérisant en outre par des peuplements floristiques et faunistiques (en particulier entomologiques) propres, ceci implique que le risque trypanosomien n'est probablement pas homogène sur l'ensemble de l'espace pastoral. Au plan vectoriel, les travaux des auteurs montrent que le campement et l'aire de repos sont quasiment dépourvus de glossines mais abondamment fréquentés par d'autres mouches piqueuses, notamment par les stomoxes. Au contraire, les populations de glossines riveraines $G$. fuscipes fus- cipes et $G$. fusca congolensis par endroits sont prédominantes au point d'abreuvement. Enfin, les sentiers et les zones de pâture peuvent être fréquentés par tous ces insectes vecteurs ainsi que par des taons $(10,11)$.

La ségrégation spatiale et temporelle des activités des animaux

Le bétail ne séjourne que très peu de temps chaque jour aux points d'abreuvements mais y revient de façon régulière. De ce fait, c'est un lieu comportant un grand risque de contamination puisque les glossines infectées y abondent. Cette observation bien établie est à l'origine de la méthode de lutte actuellement entreprise contre les trypanosomoses. Reposant sur l'emploi de pièges bipyramidaux anti-glossines $(15,16)$ disposés de façon permanente au niveau des points d'abreuvement, elle conduit à une nette amélioration de l'état sanitaire des animaux $(10,23)$. L'aire de repos est au contraire un domaine fréquenté très longuement par les zébus, pratiquement une demi-journée. Ce lieu est donc un point épidémiologiquement dangereux prévisible puisque les populations de stomoxes y abondent $(10,11)$. Hormis l'intérêt épidémiologique, la connaissance des circuits quotidiens des troupeaux peut également déboucher sur des propositions d'aménagements dans la gestion de l'espace pastoral, afin de limiter les contacts épidémiologiquement dangereux entre l'hôte et les vecteurs.

La conduite différenticlle des veaux par rapport aux adultes

Elle a, selon toute vraisemblance, un impact déterminant comme l'illustre le tableau I indiquant les taux de prévalence trypanosomienne en fonction des classes d'âge dans le cas du troupeau expérimental sédentarisé. Indépendamment de l'incertitude qui pèse encore sur la validité des résultats obtenus par la technique ELISA (puisque sa fiabilité réelle et sa standardisation sont toujours en évaluation), les résultats obtenus par les techniques microscopiques corroborent ceux issus de la technique ELISA, en traduisant de façon claire une augmentation des prévalences trypanosomiennes avec l'âge des zébus. Toutefois, l'espèce $T$. vivax fait exception, puisque les prévalences mesurées par les techniques parasitologiques sont plus élevées chez les veaux (20 p. 100) que chez les adultes de plus de trois ans $(9,3$ p. 100). Par ailleurs, l'association de toutes les techniques, stratégie actuellement préconisée pour pallier les lacunes propres à chacune des techniques employées séparément (26), montre que 55 p. 100 des veaux de moins d'un an sont affectés par au moins une espèce de trypanosome. Ces observations soulèvent au moins trois questions importantes : 1- Comment expliquer que les veaux sont moins affectés que les adultes ? 2- Comment interpréter les fortes prévalences trypanosomiennes chez les veaux âgés de moins d'un an ? 3- La transmission de $T$. vivax s'effectue-t-elle d'une manière différente des autres espèces? 
Le fait que les veaux sont moins affectés que les adultes peut découler de trois faits : une diminution progressive de la résistance vis-à-vis des trypanosomoses chez les animaux en fonction de l'âge, l'existence de traitements trypanocides plus nombreux chez les veaux ou une exposition différente des jeunes animaux au risque trypanosomien. Si l'existence de critères génétiques expliquant de manière satisfaisante l'évolution de la résistance trypanosomienne est admise, du moins pour certaines espèces de trypanosomes et de bovins, nos observations indiquent que la seconde possibilité ne peut être retenue car les veaux ne bénéficient pas systématiquement de traitements trypanocides plus nombreux que les adultes. Nous préférons donc retenir la troisième hypothèse, objet du présent article. Elle est basée sur le fait que des pratiques pastorales différentes selon l'âge des animaux entraînent une exposition au risque trypanosomien différente.

Cependant, cette explication se heurte à un obstacle majeur : comment interpréter les fortes prévalences trypanosomiennes chez les veaux de moins d'un an alors qu'ils sont soustraits de la pression trypanosomienne liée aux glossines riveraines ? En effet, jusqu'à l'âge de 6 mois, ils restent attachés en permanence à l'aire de repos, tandis que les mois suivants, ils ne fréquentent que rarement et irrégulièrement les abreuvoirs au cours de leur circuit quotidien peu éloigné du campement (figure 3). Ces considérations nous amènent à adopter un schéma épidémiologique plus complexe que celui admis jusqu'à présent. Ce schéma encore hypothétique confirmerait le rôle vecteur majeur de $G$. $f$. fuscipes dans la transmission aux bovins des trois espèces de trypanosomes tout en impliquant de façon plus concrète d'autres insectes hématophages, vecteurs accessoires $(10,11)$. Dans la zone étudiée, les stomoxes sont les candidats les plus sérieux dans la transmission de certains trypanosomes en particulier de $T$. vivax (10). En raison des circuits de pâture des zébus et de l'écologie des insectes évoqués, les bovins adultes sont essentiellement soumis à la pression trypanosomienne de $G$. $f$. fuscipes tandis que les veaux sont surtout exposés aux piqûres des stomoxes. Ceci n'exclut pas cependant que les adultes soient fréquemment piqués par les stomoxes lors de leur séjour quotidien à l'aire de repos. II est communément admis que les glossines, en assurant une transmission cyclique des trypanosomes entraînant une multiplication importante de ceux-ci à l'intérieur de la glossine, sont des vecteurs très efficaces de ces agents tandis que les stomoxes, en transmettant certains trypanosomes par voie mécanique uniquement ont une compétence vectorielle plus atténuée. De ce fait, il n'est pas impossible que les prévalences trypanosomiennes plus élevées chez les zébus adultes soient tout simplement le reflet de ces différences de compétence vectorielle, elles-mêmes favorisées par des pratiques pastorales différentes chez les veaux et chez les adultes.

\section{Variabilité des pratiques pastorales et diversité épidémiologique}

Cette étude souligne le rôle des pratiques pastorales en tant que facteur épidémiologique. II est donc utile d'attirer l'attention sur l'existence de nombreux autres facteurs ayant une action directe sur les pratiques pastorales et par conséquent des retombées épidémiologiques importantes.

Parmi ces facteurs figure en premier lieu le facteur humain. Les stratégies de l'éleveur interviennent beaucoup sur les pratiques de conduite du troupeau. Tous les cas de figures existent, de l'éleveur qui, sensible à l'attrait de l'herbe nouvelle pour son troupeau ou pour des raisons personnelles, pratique une transhumance régulière à l'éleveur qui n'hésite pas à contraindre ses bêtes à une certaine immobilité durant la saison sèche. En règle générale, le comportement des Peul Mbororo en Centrafrique peut se concrétiser à tout moment par un changement rapide et imprévisible des habitudes pastorales d'un éleveur. La conséquence directe est une modification immédiate des circuits quotidiens des zébus et donc du risque trypanosomien.

TABLEAL I

Prévalences trypanosomiennes dans les diverses classes d'âge chez les zébus. Comparaison des résultats obtenus avec les méthodes microscopiques (frottis, goutte épaisse et concentration en tubes capillaire) et immunodiagnostique (ELISA).

Les prévalences globales (toutes espèces confondues) sont obtenues en associant toutes les méthodes de détection des trypanosomes.

Prévalences par espèces (p. 100)

Technique ELISA

Prévalences
globales
(p. 100)

T. b. brucei T. vivax

T. congolense

n Trypanosomaspp.

n T. b. brucei T. vivax T. congolense$$
\text { n }
$$

\begin{tabular}{|c|c|c|c|c|}
\hline $\begin{array}{l}-1 \text { an } \\
-3 \text { ans } \\
3 \text { ans }\end{array}$ & $\begin{array}{r}80 \\
152 \\
247\end{array}$ & $\begin{array}{l}0 \\
1,97 \\
0,4\end{array}$ & $\begin{array}{l}20 \\
17,8 \\
9,3\end{array}$ & $\begin{array}{l}0 \\
1,3 \\
6,1\end{array}$ \\
\hline
\end{tabular}

= effectif par classe d'âge.

\section{1}

152

247

24,7

30,3

12,4

31,6
80

150

245

475
55

64

77,1
50,7

64,8 


\section{F. D'Amico J.M. Poussinga C. Le Masson A. Le Masson D. Cuisance}

Les facteurs liés à la physiologie des zébus sont également importants. Les zébus apprécient l'alimentation complémentaire en natron ou en sel que leur fournit l'éleveur. En cas d'approvisionnement insuffisant de la part de celui-ci, la carence de ces éléments pousse les zébus à modifier leur comportement de pâture et leurs circuits quotidiens. Le troupeau peut refuser de partir au pâturage ou bien, s'il y est chassé par l'éleveur, peut ne pas rentrer au campement pendant quelques jours et errer loin dans la savane (dans de nouveaux espaces épidémiologiques) à la recherche de substituts de ces composés. A ces considérations physiologiques, il faut ajouter les perturbations comportementales apparaissant chez les taureaux et chez les vaches gestantes. Toutefois, le principal stress physiologique imposé au bétail réside dans le nombre élevé d'infections parasitaires récurrentes. En Centrafrique, Bouchet et al. (4) montrent que les veaux en particulier sont très exposés à ce polyparasitisme dû surtout à des parasites sanguins et gastro-intestinaux. II est par ailleurs supposé que toutes ces causes de stress favorisent l'installation des trypanosomoses et en augmentent les conséquences pathologiques.

L'impact des facteurs liés à la qualité et à la quantité de fourrage disponibles n'est pas négligeable. L'état du pâturage préside largement aux déplacements des zébus puisqu'une herbe devenue rare ou bien trop haute est refusée par les animaux ce qui les incite à quitter les lieux. L'invasion du pâturage par l'herbe du Laos Chromolaena odorata (en pleine expansion aujourd'hui en RCA (17)) peut constituer également une cause majeure d'abandon par les zébus. Par ailleurs, en saison des pluies, le bétail réduit son parcours pour se concentrer sur les zones déjà broutées et riches en repousses très appréciées, et délaisse les secteurs composés de végétaux moins appétents qui commencent leur montaison.

Enfin les facteurs physiques peuvent avoir d'importantes répercussions. Ainsi, pour les élevages installés sur les bombements latéritiques imperméables (bowé), l'existence en saison des pluies de petites retenues d'eau conduit souvent le troupeau à s'y abreuver ; il ne descend plus vers la galerie forestière où séjournent les glossines et devient donc moins exposé à leur pression vectorielle. De même, les violentes précipitations survenant surtout de juillet à septembre et leurs conséquences modifient le circuit quotidien du bétail. Les rivières en crue barrant la route habituelle du pâturage, le troupeau change d'itinéraire ou attend le retour de conditions plus clémentes (figure 3). De façon opposée, les années de sécheresse conduisent à l'assèchement total de certains points d'eau pendant la saison sèche. Dans ces conditions, l'éleveur est souvent obligé de modifier son circuit de transhumance, de déplacer son campement de saison sèche. Les zébus, quant à eux, peuvent répondre à ces perturbations en allongeant leurs circuits quotidiens. Toutes ces modifications conduisent à un bouleversement sensible du risque trypanosomien, par soustraction à la pression vectorielle habituelle ou au contraire par addition à celle-ci.

\section{CONCLUSION}

\section{Pour une approche pluridisciplinaire et de nouvelles techniques d'investigation}

A l'heure actuelle, contrairement à ce qui se passait il y a une trentaine d'années $(12,32)$, les Peul Mbororo connaissent une aggravation de leur situation, se traduisant par un appauvrissement en bétail (3). En dépit du fait que les espaces libres en Centrafrique restent relativement importants, des contraintes d'ordre sanitaire principalement (trypanosomoses, maladies transmises par tiques et streptothrichose), mais aussi socio-économique, dissuadent les Mbororo d'adopter la solution qui leur était chère : la fuite. De nombreux éleveurs sont obligés de changer de statut, en évoluant vers une certaine forme de sédentarité. Dans la zone considérée, 54 p. 100 d'éleveurs se sont mis maintenant à cultiver, et leur nombre s'accroît rapidement (25). Par ailleurs, ils sont déjà nombreux à élever des moutons (76 p. 100 des familles) et des chèvres (12 p. 100). A l'échelle du pays, ces changements se traduisent par une augmentation sensible de la durée moyenne d'établissement d'un campement d'éleveurs, celle-ci passant en une dizaine d'années de 7,8 à 8,7 ans (25). Cette évolution ne convient pas forcément aux zébus qui doivent pâturer de plus en plus loin en saison sèche, ce qui multiplie les rencontres avec d'autres troupeaux et les dégâts dans les champs cultivés. Le risque de conflits est ainsi augmenté dans un milieu pastoral déjà fortement chargé et souvent dégradé par endroits $(1,17)$.

Une observation fine et à grande échelle de l'espace pastoral Mbororo montre que son utilisation est relativement organisée et qu'elle évolue au cours de l'année. Une analyse synthétique de ce facteur pastoral avec d'une part les variations d'abondance et la dynamique de distribution des glossines et des autres insectes piqueurs vecteurs potentiels de trypanosomoses $(10,11)$ et, d'autre part leur taux d'infection trypanosomienne, permettra de définir la notion "d'espace épidémiologique trypanosomien". A long terme, en intégrant étroitement la circulation des trypanosomes et l'activité spatio-temporelle du bétail, une approche plus complète de la modélisation de l'épidémiologie des trypanosomoses $(33,34)$ pourra être envisagée. Cependant, la grande richesse de situations rencontrées dans les systèmes d'élevage et la complexité fonctionnelle du "couple vecteur/parasite" restent des obstacles difficiles à surmonter. Toutefois, les résultats du présent travail permettent déjà d'envisager le fonctionnement du système épidémiologique trypanosomien sous un angle nouveau (10). Au-delà de ces considérations, le propos essentiel de ce travail est de souligner l'importance des pratiques pastorales en tant que facteur épidémiologique, aspect jusqu'à présent trop souvent négligé.

La nécessité de développer des enquêtes portant sur de longues périodes et visant à mieux comprendre la diversité des situations d'élevage et des modes de conduite du bétail a déjà été soulignée (7). Dans l'avenir, des études multidisciplinaires faisant appel à diverses méthodologies empruntées à la géographie, la cartographie, 
l'ethnologie ou l'informatique devraient être généralisées et appliquées à la quantification de la distribution spatiale et temporelle de "l'activité" des différentes catégories de bovins au sein de cet espace pastoral. Et pour pouvoir mieux suivre les déplacements des zébus dans les hautes herbes de Centrafrique, ce bétail très mobile dont l'éthologie est très dépendante de la qualité de l'herbe, on peut se poser la question de l'extension de l'usage des techniques de télémétrie et des systèmes d'information géographique (S.I.G.).

Outre l'apport fondamental à la connaissance de ce milieu Mbororo en Centrafrique, de telles études devraient contribuer efficacement à la connaissance de l'épidémiologie des trypanosomoses bovines mais aussi d'autres parasitoses également contraignantes pour le développement de l'élevage comme les helminthoses et surtout les maladies transmises par les tiques. Ces travaux apportent d'intéressantes perspectives de collaboration avec l'éleveur qui devrait pouvoir en tirer quelques éléments utiles à l'amélioration de l'état sanitaire de son cheptel.

\section{Remerciements}

Nous tenons à remercier tout particulièrement le $\mathrm{Dr} \mathrm{J}$. Desrotour, responsable du Service de l'Elevage en RCA pendant de longues années, pour ses précieux conseils sur les Mbororo et leurs pratiques pastorales. Ce manuscrit a bénéficié des remarques et commentaires de $\mathrm{Ph}$. Lhoste, de E. Landais et de J. Boutrais qui en outre s'est chargé de la transcription correcte des noms en fulfuldé. Nous sommes redevables également à Messieurs D. Demba, F. N'Dokoué, E. Pounékrouzou, J.P. Gouteux, F. Blanc, F. Le Gall, P. Cailton, J.M. Guillerme et M. Mainguet pour leur aide sur le terrain. Enfin, nous ne saurions oublier l'Agence nationale pour le développement de l'élevage en RCA (ANDE), ainsi que son Directeur Général, M. A. Kota-Guinza, qui ont grandement facilité notre recherche, notamment en nous permettant de travailler sur leur troupeau expérimental.

\section{Bibliographie}

1. AUDRU J., HEDIN P., 1971. Bilan des études agrostologiques en République Centrafricaine. Maisons-Alfort, France, I.EM.V.T., 8 p. (document multigraphié)

2. BLANC F., 1991. Lutte antiglossinaire en République Centrafricaine. Montpellier, France, C.N.E.A.R.C., 118 p. (rapport stage ESAT 1)

3. BLANC F., LE MASSON C., ASSANA REMAYEKO, LE MASSON A., LE GALL F., LHOSTE P., 1992. Les raisons d'un engagement agricole irréversible des éleveurs Mbororo de République Centrafricaine. Cah Rech. Dév., 32 : 6-18.

4. BOUCHET A., GRABER M., FINELLE P., DESROTOUR J., MACON G., 1969. Le parasitisme du zébu dans l'ouest de la République Centrafricaine. I. Parasitisme des veaux de lait. Revue Elev. Méd. vét. Fays trop., 22 (3) : $373-383$

5. BOITTRAIS I., 1988. Des Peuls en savanes humides. Développement pastoral dans l'Ouest Centrafricain. Paris, France, ORSTOM Editions. (coll. Etudes et Thèses)

6. BOUTRAIS J., 1990. Les savanes humides, dernier refuge pastoral : l'exemple des WodaaBe de Centrafrique. Genève-Afrique, 28 (1) : 65-90.

7. CUISANCE D., 1987. Compte rendu de situation et propositions de pro- gramme. Rapport de mission d'appui à l'Unité de Lutte contre les Glossines dans le cadre du Projet National de Développement de l'Elevage. Ministère du Développement Rural, République Centrafricaine. MaisonsAlfort, France, IEMVT, 65 p.

8 CUISANCE D CAILTON P KOTA-GUINZA A N'DOKOUÉ F POUNÉKROZOU E., DEMBA D., 1991. Lutte contre Glossina fuscipes fuscipes par piégeage chez les éleveurs Mbororo de République Centrafricaine. Revue Élev. Méd. vét. Pays trop., 44 (1) : 81-89.

9. CUISANCE D., GOUTEUX J.P., CAILTON P., KOTA-GUINZA A N'DOKOUÉ F., POUNÉKROZOU E., DEMBA D., 1992. Problématique d'une lutte contre les glossines pour la protection de l'élevage zébu en République Centrafricaine. Mém. Soc. r. belge Ent., $35: 103-110$.

10. D'AMICO F., 1993. Rôle de Glossina fuscipes fuscipes Newstead, 1910 dans la transmission des trypanosomoses bovines en Afrique centrale. Le cas de la zone d'élevage d'Ouro-Djafoun (République Centrafricaine). Thèse doct., Université Montpellier II, France, $267 \mathrm{p}$

11. D'AMICO F., GOUTEUX J.P., CUISANCE D., MAINGUET M. POUNÉKROZOU E., N'DOKOUÉ F., LE GALL F., 1992. Epidémiologie des trypanosomoses bovines en République Centrafricaine : évaluation de l'importance de la vection mécanique. In: Premier séminaire international sur les trypanosomoses animales non transmises par les glossines, Annecy, France, 14-16 octobre 1992.

12. DESROTOUR J., 1967. Les pasteurs Bororo et leurs troupeaux. Organisation. Modes de vie. Modes d'élevage. In : Lacrouts M., Samiguet J. Tyc J. eds, Le cheptel bovin de la République Centrafricaine. Production, commercialisation, perspectives d'avenir. Paris, France, Secrétariat d'Etat aux Affaires Etrangères chargé de la Coopération (République française) Bangui, RCA, Ministère du Développement (République Centrafricaine) p. 287-297.

13. FINELLE P., 1957. Les trypanosomoses bovines dans l'ouest de l'Oubangui-Chari. Essai de traitement par le bérénil. Revue Élev. Méd. vét Pays trop., $10: 231-247$.

14. FRANQUIN P., DIZIAIN R., COINTEPAS J.P., BOULVERT Y, 1988. Agroclimatologie du Centrafrique. Paris, France, ORSTOM éditions. (collection Initiations, Documentations techniques il" 71)

15. GOUTEUX J.P., 1991. La lutte par piégeage contre Glossina fuscipes fuscipes' pour la protection de l'élevage en République centrafricaine. II Caractéristiques du piège bipyramidal. Revue Élev. Méd. vét. Pays trop., 44 (3) : 295-299

16. GOUTEUX J.P., CUISANCE D., DEMBA D., N'DOKOUÉ F., LE GALL F., 1991. La lutte par piégeage contre Glossina fuscipes fuscipes pour la protection de l'élevage en République centrafricaine. I. Mise au point d'un piège adapté à un milieu d'éleveurs semi-nomades. Revue Élev. Méd. vét. Pays trop., 44 (3) : 287-294.

17. HUGUENIN J., BEDOGO B., 1990. Projet de préservation des pâtu rages des régions Est de la République centrafricaine contre l'envahissement par Chromolaena odorata. Bangui, RCA, ANDE, Service de Malherbologie agropastorale, $18 \mathrm{p}$. (rapport)

18. ITARD J., 1981. Les trypanosomoses animales africaines. In : Préci de parasitologie vétérinaire tropicale. Tome II. Paris, France, Ministère de la Coopération et du Développement, p. 303-369. (coll. Manuels et Précis d'élevage, 10 )

19. KINTZ D., 1988. Les éleveurs centrafricains. Leurs caractéristiques sociologiques et linguistiques. Bangui, RCA, Projet National de Développement de l'Élevage, 89 p. (rapport)

20. KOTA-GUINZA A., 1986. Mise en place des pharmacies vétérinaires villageoises et analyse de l'activité des premiers groupements d'intérêt pastoraux de la République Centrafricaine. Maisons-Alfort. France, IEMVT, 64 p. (mémoire DESS)

21. LEAK S.G.A., AWOME K., COLARDELLE C., DUFFERA W. FERON A., MAHAMET B., MAWUENA K., MINENGU M., MULUN GO M., NANKODABA G., ORDNER G., PELO M., SHERIA M., TIKUBET G., TOURE M., YANGARI G., 1988. Determination of tsetse challenge and its relationship with trypanosome prevalence in trypanotolerant livestock at sites of the African Trypanotolerance Livestock Network. In Livestock Production in Tsetse Affected Areas of $\Lambda$ frica, Proc. Meet., Nai 


\section{F. D'Amico J.M. Poussinga C. Le Masson A. Le Masson D. Cuisance}

robi, Kenya, 23rd-27th November 1987. Nairobi, Kenya, ILCA/ILRAD, p. 43-54.

22. LE GALL F., N'DOKOUÉ F., MAINGUET M., 1992. Résultats d'une enquête large réalisée sur 27 secteurs d'élevage en RCA (1991) : maladies transmises par les tiques et trypanosomoses. Espèces vectrices, coûts des mortalités et traitements. Bangui, RC.A, DSARA/ANDE. (rapport)

23. LE GALL F., BLANC F., GOUTEUX J.P., MAINGUET M., CUISANCE D., LEMESRE J.L., NITCHEMAN S., CAVALEYRA M., D'AMICO F., POUNÉKROZOU E., N'DOKOUÉ F., 1995. La lutte par piégeage contre Glossina fuscipes fuscipes pour la portection de l'élevage en République centrafricaine. IV. Impact entomologique, parasitologique et zootcchniquc. Revue Élev. Méd. vét. Pays trop., 48 (2) :161-169.

24. LE MASSON C., 1985. Les éleveurs Mbororo de l'Ombella-M'Poko. Approvisionnement vivrier - Budget familial - Commerce des produits laitiers. Bangui, RCA, Ministère du Développement Rural, Projet de Développement de la Région Ouest, $75 \mathrm{p}$.

25. LE MASSON C., REMAYEKO A., 1990. Les éleveurs Mbororo étude socio-économique. Bangui, RCA, Agence Nationale de Développement de l'Elevage, 227 p. (rapport)

26. LUCKINS A.G., 1992. Methods for diagnosis of trypanosomiasis in livestock. Wld Anim. Rev., 70/71: 15-20.

27. MATHIEU R., 1988. Mode d'utilisation des pâturages de saison des pluies par les éleveurs Mbororo dans la région de Bossembélé (République centrafricaine). Toulouse, France, ESAP, 57 p. + annexes. (rapport de stage)

28. MURRAY M., MURRAY P.K., McINTYRE W.I.M., 1977. An improved parasitological technique for the diagnosis of african trypanosomiasis Trans. R. Soc. trop. Med. Hyg., 71: 325-326.

29. NANKODABA G., COULIBALY L., HECKER P., LEAK S.G.A., SCHUETTERLE A 1988. Trypanosome prevalence in cattle herds exposed to a range of tsetse challenge levels in northern Côte d'Ivoire. In: Livestock Production in Tsetse Affected Areas of Africa, Proc. Meet., Nairobi Kenya, 23rd-27th November 1987. Nairobi, Kenya, ILCA/ILRAD, p. 5569.

30. NANTULYA V M. MUSOKE A., RURANGIRWA F.R., SAIGAR N., MINJA S.H., 1987. Monoclonal antibodics that distinguish Trypanosoma congolense, T. vivax and T. brucei. Parasit. Immun., 9: 421-431.

31. NANTULYA V.M., LINDQVIST K.J., STEVENSON P., MWANGI E.K., 1992, Application of a monoclonal antibody-based antigen detection

D'AMICO (F.), POUSSINGA (J.M.), LE MASSON (C.), LE MAS SON (A.), CUISANCE (D.). Mbororo pastoral practices and bovine trypanosomosis in an area of wet savannah of the Central African Republic. Revue Élev. Méd. vét. Puys trop., 1995, 48 (2) : 203-212

A study was undertaken to understand i) the organization of the pastoral space of the Mbororo cattle breeders in the Central $\Delta$ frican Republic and ii) the spatial movements of their zebu cattle inside this space. Besides the breeder's encampment, the pastoral space is divided into three main areas: the cattle rest area, the cattle's watering place and the pasture which is criss-crossed by numerous paths. This investigation reveals that the use of the pastoral space is based on a spatial and temporal segregation of the movements of the zebu cattle. In the wet savannahs of the Central African Republic where Glossina fuscipes fuscipes Newst. 1910 is the main vector of bovine trypanosomosis the authors emphasize that a thorough examination of the pastoral strategies provides further elements for a comprehensive understanding of the epidemiology of nagana. For example, the differential management of calves and adult cattle is probably an important epidemiological factor.

Key words : Cattle - Zebu cattle - Trypanosomosis - Glossina fuscipes fuscipes - Epidemiology - Pastoralism - Pastoral society - Rangelands Transhumance - Central African Republic. enzyme-linked immunosorbent assay antigen (ELISA) for field diagnosis of bovine trypanosomiasis at Nguruman, Kenya. Ann. trop. Med. Parasit. 86 (3): $225-230$

32. PRIOUL C., 1971. Eleveurs nomades et paysans sédentaires dans le nord-ouest centrafricain. Trav. Docum. Géogr, trop. , $3: 1-42$.

33. RAWLINGS P., DWINGER R.H., SNOW W.F., 1991. An analysis of survey measurements of tsetse challenge to trypanotolerant cattle in relation to aspects of analytical models of trypanosomiasis. Parasitology 102 $371-377$

34. RAWLINGS P., WACHER T.J., SNOW W.F., 1994. Cattle-tsetse contact in relation to the daily activity patterns of Glossina morsitans submorsitans in The Gambia. Med. vet. Ent., 8 (1): 57-62.

35. ROGERS D.J., 1985. Trypanosomiasis "risk" or "challenge": a review Acta trop., 42: 5-23.

36. ROWLANDS G.J., WOUDYALEW MULATU, AUTHIÉ E., D'IETEREN G.D.M LEAK S.G A NAGDA S.M. PEREGRINE A.S., 1993 Epidemiology of bovine trypanosomiasis in the Ghibe valley, southwest Ethiopia. 2. Factors associated with variations in trypanosome prevalence, incidence of new infections and prevalence of recurrent infections. Acta trop., 53: $135-150$

37. SILLANS R., 1958. Les savanes de l'Afrique Centrale. Paris, France, Lechevalier

38. SNOW W.F., RAWLINGS P., CLAXTON J.R., MILLIGAN P.J. 1989. Facets of challenge. In: 25th British Society of Parasitology Trypanosomiasis Seminar, Glasgow, United Kingdom,13-15 September 1989

39. WACHER T.J., 1989. Host movement and population dynamics of tsetse flies. I $n$ : 25th British Society of Parasitology Trypanosomiasis Seminar. Glasgow, United Kingdom, 13-15 September 1989.

40. WACHER T., RAWLINGS P., JEANIN P., 1988. Glossine et trypanosomes chez les bovins : leurs rapports avec les régimes de pâturage et leur importance I $n$. Livestock Production in Tsetse Affected Areas of Africa. Proc. Meet., Nairobi, Kenya, 23rd-27th November 1987. Nairobi, Kenya, ILCA/ILRAD, p. 78-81.

41. YVORÉ P., DESROTOUR J, LAURENT J., FINELLE P., 1962. Essai d'assainissement d'une zone infestée par Glossina fuscipes fuscipes Newstead en République centrafricaine. Revue Élev. Méd, vét. Pays trop., 15 (4) : 403-410

D'AMICO (F.), POUSSINGA (J.M.), LE MASSON (C.), LE MAS SON (A.), CUISANCE (D.). Prácticas de pastoreo Mbororo y tripanosomosis bovinas en una zona de savanas húmedas de República centroafricana.Revue Élev. Méd. vét. Pays trop., 1995, 48 (2) : 203-212

El estudio se llevó a cabo sobre la distribución del espacio pastoril por parte de los criadores Mbororo de República centroafricana, así como los movimientos de los cebúes rojos. Además del campamento de los criadores, este espacio está dividido en tres partes principales : área de reposa, abrevadero y pastizal atravesado de senderos. La utilisación reposa sobre una segregación espacial y temporal de los desplazamientos de los animales. En el contexto particular de las savvanes húmedas del centro de la República centroafricana, en donde la especie Glosisina fuscipes fuscipes Newst. 1910 es el vector principal de la tripanosomosis bovinas, los autores demuestran que el conocimiento profundo de las prácticas pastoriles, aporta nuevos elementos a la comprensión de la epidemiología del nagana. De esta manera, el comportamiento diferencial de los terneros con respecto a los adultos es aparentemente un factor epidemiológico capital.

Palabras clave : Bovino - Cebú - Tripanosomosis - Glossina fuscipes fuscipes - Epidemiología - Pastoralismo - Sociedad pastoral - Tierras de pastos - Trashumancia - República centroafricana. 